\title{
3D Imaging of Water Behavior at Millimeter Wavelength
}

\author{
A. SAYinti*, A. KAYA And A. Vertiy \\ Material Institute, TUBITAK-MRC, Gebze, Kocaeli, Turkey
}

\begin{abstract}
During the transmission of electromagnetic radiation through a medium containing water molecules, portions of the electromagnetic spectrum are absorbed by water molecules. This water absorption occurs preferentially at certain characteristic wavelengths while the balance of the spectrum is transmitted with minimal effects. This characteristic of water provides advantages but also some disadvantages in science and engineering applications. Especially in remote sensing applications, it may cause to produce incorrect information. So identification of behavior of electromagnetic wave in water is significantly important. Some of the studies based on electromagnetic wave behavior in water are bio-medical researches, sensors, transmitters, accumulators, organic-inorganic materials, and microwave ovens. In this paper, based on the water's absorption of electronic wave property, a plastic cup as half-full of water is imaged using near field microwave system. After being processed with 3D-DLL algorithm it is imaged as 3D with IRIS software. A robotic system is used for data collection process. Transmission and reflection measurements of system are done with N5230A network analyzer.
\end{abstract}

DOI: 10.12693/APhysPolA.123.467

PACS: $84.40 .-\mathrm{x}$

\section{Introduction}

Water is an important substance in every aspect of life. However, many physical properties and processes of water are not well understood; as such it has been and will continue to be a focus of extensive investigation [1]. During the transmission of electromagnetic radiation through a medium containing water molecules, portions of the electromagnetic spectrum are absorbed by water molecules. This water absorption occurs preferentially at certain characteristic wavelengths while the balance of the spectrum is transmitted with minimal effects [2].

In this study, to observe the behavior of water at millimeter wavelength, imaging of water in plastic and PTFE cup via microwave tomography method is examined. Results are shown as 2D and 3D below.

\section{Experimental}

Measurement system consists of antennas, linear scanner driver, and control computer. Experimental system can be seen at Fig. 1.

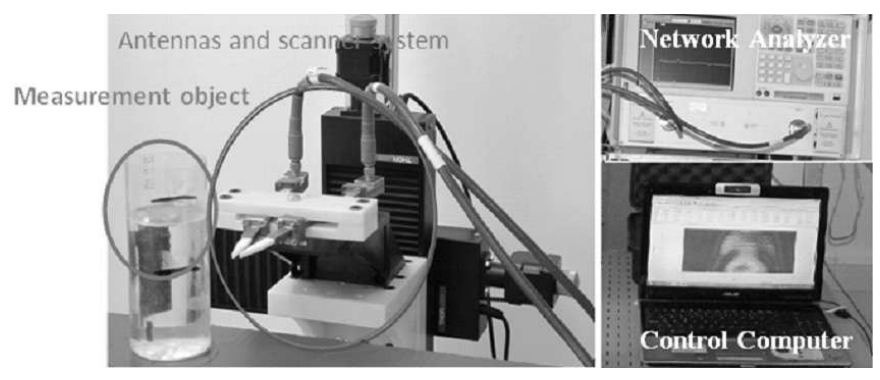

Fig. 1. Millimeter wave band tomography system.

${ }^{*}$ corresponding author; e-mail: aysun.sayinti@tubitak.gov.tr
Tomography algorithm is shown below. It is assumed that the depolarization is negligible. If polarization current is normalized by incident field, the scattered field can be presented in the form of

$$
\begin{aligned}
& \psi(x, y, z)=k^{2} \iiint_{V} E_{z}^{i}\left(\theta^{i}, x^{\prime}, y^{\prime}\right) K\left(x^{\prime}, y^{\prime}, z^{\prime}\right) \\
& \quad \times G\left(x, y, z ; x^{\prime}, y^{\prime}, z^{\prime}\right) \mathrm{d} x^{\prime} \mathrm{d} y^{\prime} \mathrm{d} z^{\prime},
\end{aligned}
$$

where $G\left(x, y, z ; x^{\prime}, y^{\prime}, z^{\prime}\right)$ is free space Green function, $K\left(x^{\prime}, y^{\prime}, z^{\prime}\right)$ is a normalized polarization current and $E_{z}^{i}\left(\theta^{i}, x, y\right)=\mathrm{e}^{\mathrm{i} k\left(x \sin \theta^{i}+y \cos \theta^{i}\right)}$ is an incident plane wave. Let us define the two-dimensional Fourier transform $\hat{\psi}\left(v_{1}, y, v_{3}\right)$ of the scattered field $\psi(x, y, z)$ on a scanning plane at $y=$ const and also the three-dimensional Fourier transform $\hat{K}(\alpha, \beta, \chi)$ of $K(x, y, z)$. Applying the two-dimensional Fourier transform of scattered field $\psi(x, y, z)$ for (1), one can obtain the following equation:

$$
\begin{aligned}
\hat{\psi} & \left(v_{1}, y, v_{2}\right)=k^{2} \int_{-\infty}^{\infty} \int_{-\infty}^{\infty} \int_{-\infty}^{\infty} \mathrm{e}^{\mathrm{i} k\left(x^{\prime} \sin \theta^{i}+y^{\prime} \cos \theta^{i}\right)} \\
& \times K\left(x^{\prime}, y^{\prime}, z^{\prime}\right) \\
& \times \underbrace{\frac{\mathrm{i}}{2} \frac{\mathrm{e}^{-2 \pi v_{1} x^{\prime}} \mathrm{e}^{\mathrm{i} \gamma\left(y^{\prime}-y\right)} \mathrm{e}^{-2 \pi \mathrm{i} v_{3} z^{\prime}}}{\gamma}}_{g\left(v_{1}, y, v_{3} ; x^{\prime}, y^{\prime}, z^{\prime}\right)} \mathrm{d} x^{\prime} \mathrm{d} y^{\prime} \mathrm{d} z^{\prime},
\end{aligned}
$$

where $g\left(v_{1}, y, v_{3} ; x^{\prime}, y^{\prime}, z^{\prime}\right)$ is the two-dimensional Fourier transform of the Green function, and $\gamma=2 \pi v_{2}=$ $\sqrt{k^{2}-4 \pi^{2}\left(v_{1}^{2}+v_{3}^{2}\right)}$ is the wave number. If one rearranges Eq. (2), it becomes

$$
\hat{\psi}\left(v_{1}, y, v_{2}\right)=\frac{\mathrm{i}}{2 \gamma} \mathrm{e}^{-\mathrm{i} \gamma y} k^{2}
$$




$$
\times \underbrace{\int_{-\infty}^{\infty} \int_{-\infty}^{\infty} \int_{-\infty}^{\infty} \frac{K\left(x^{\prime}, y^{\prime}, z^{\prime}\right)}{\gamma} \exp (-2 \pi \mathrm{i} x^{\prime} \underbrace{\left(v_{1}-\frac{k \sin \theta^{i}}{2 \pi}\right)}_{\alpha}-2 \pi \mathrm{i} y^{\prime} \underbrace{\left(\frac{\gamma+k \cos \theta^{i}}{2 \pi}\right)}_{\beta}-2 \pi \mathrm{i} \underbrace{v_{3}}_{\gamma} z^{z^{\prime}}) \mathrm{d} x^{\prime} \mathrm{d} y^{\prime} \mathrm{d} z^{\prime} .}_{\hat{K}(\alpha, \beta, \gamma)}
$$

Equation (3) can be rewritten as

$$
\hat{K}(\alpha, \beta, \chi)=\hat{\psi}\left(v_{1}, y, v_{3}\right) \frac{2 \gamma}{k^{2} \mathrm{i} \mathrm{e}^{-\mathrm{i} \gamma y}} .
$$

Finally, by taking the inverse Fourier transform of $\hat{K}(\alpha, \beta, \chi)$, the object function $K(x, y, z)$ is obtained [3-5].

\section{Scanner system}

Scanner system is the most important step in a measurement. The operator should choose the correct specifications, such as system speed, acceleration, trigger points, in order to get proper scan data. To be able to achieve a surface scan, two-dimensional, $2 \mathrm{D}$, scan is needed. One dimension is assigned to scan the object horizontally while other moves the first axis vertically along the object. Each axis should follow a motion curve in order not to speed up immediately. Figure 2 shows a motion curve.

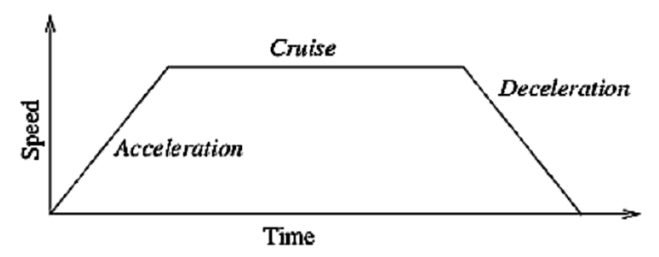

Fig. 2. Speed of a motion.

In Fig. 2, speed profile for a motion can be seen. The curve is divided into three segments: acceleration, cruise and deceleration. The level of the cruise phase is given by the speed setting of the motion, while the steepness of the acceleration and deceleration phases is given by the acceleration parameter.

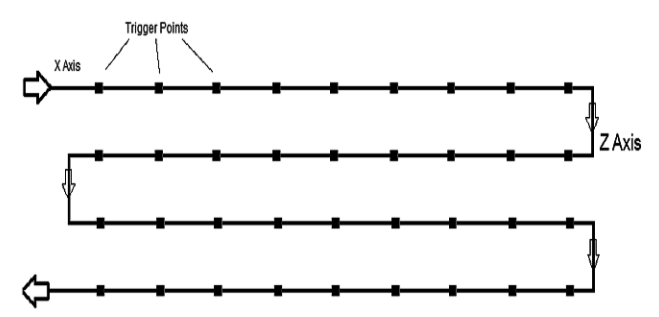

Fig. 3. Axis movements.

As it can be seen in Fig. 3, scan axis is divided by trigger points on which data is requested. Data on trigger points is collected on the operator's computer. After scan is finished, raw data is given to tomography algorithm.

\section{Results and discussion}

In the table, measurement data on water in the plastic cup and in the teflon cup are given. In Figs. 4-6 the tomographic images are shown.

Measurement data.

TABLE

\begin{tabular}{c|c|c}
\hline \hline Object type & Frequency range & Scan area \\
\hline water in the plastic cup & $30-35 \mathrm{GHz}$ & $10 \times 5 \mathrm{~cm}^{2}$ \\
water in the teflon cup & $30-35 \mathrm{GHz}$ & $7 \times 7 \mathrm{~cm}^{2}$
\end{tabular}

2D and 3D images of water in two different levels, which are contained by $50 \mathrm{~mm}$ diameter and $0.05 \mathrm{~mm}$ thick plastic cup, is shown in Figs. 4 and 5.

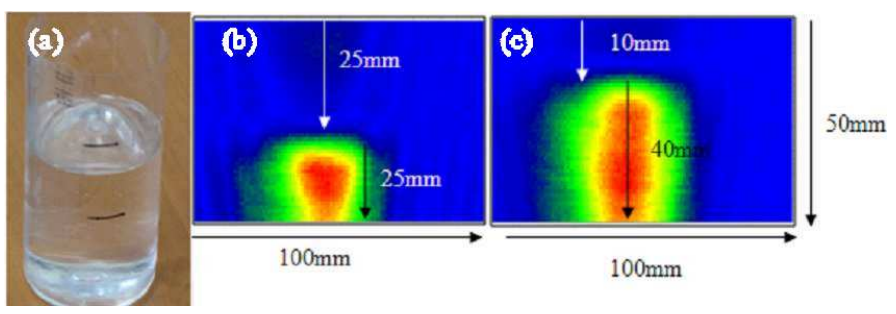

Fig. 4. 2D tomographic surface image of the plastic glass. Scan of $10 \times 5 \mathrm{~cm}^{2}$ at depth $z=2 \mathrm{~mm}$ : (a) photo of the plastic cup with water, (b) - 2D scanned image at water height $25 \mathrm{~mm},(\mathrm{c})-2 \mathrm{D}$ scanned image at water height $40 \mathrm{~mm}$.
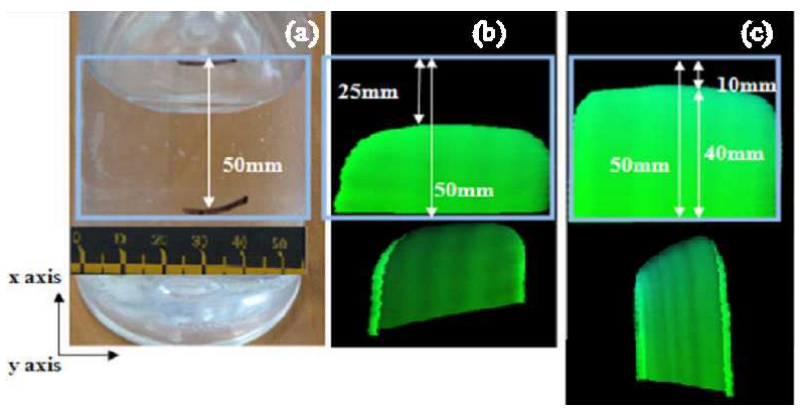

Fig. 5. 3D tomographic images, as in Fig. 4.

The $2 \mathrm{D}$ image of water which is contained by $55 \mathrm{~mm}$ diameter and $20 \mathrm{~mm}$ thick teflon glass is shown in Fig. 6 . 3D image is shown in Fig. 7. A metal marker is used in order to define the start point of scan. 

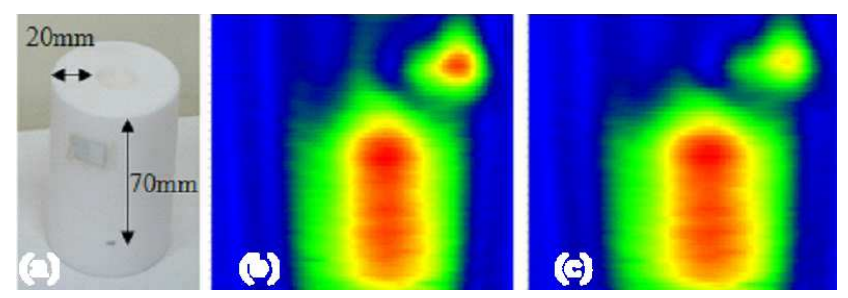

Fig. 6. 2D tomographic surface image of the teflon cup with water height level of $43 \mathrm{~mm}$, with surface scan area in $x-y=7 \times 7 \mathrm{~cm}^{2}$. (a) - photo of the teflon cup plastic cup with water, (b) - 2D scanned image at depth $z=$ $7 \mathrm{~mm},(\mathrm{c})-2 \mathrm{D}$ scanned image at depth $z=40 \mathrm{~mm}$.
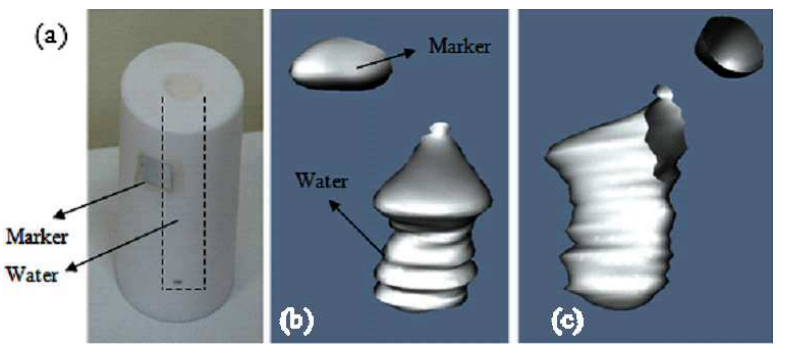

Fig. 7. 3D tomographic images, as in Fig. 6.

\section{Conclusions}

In this work, between 30-35 GHz frequency band, the behavior of water which is in the two different glasses of different thicknesses and diameters are studied. After scanning both glasses in the frequencies mentioned above, thanks to tomography algorithm, differences between real size of glass and the size of processed output are compared. In terms of dimensions, it is observed that processed image is compatible with the actual glass.

Because of glass thickness, processed image of water filled plastic glass has much smoother image compared to the teflon glass.

\section{Acknowledgments}

In memory of Sergiy Gavrilov.

\section{References}

[1] J.R. Peacock, Millimeter wave permittivity of water near $25^{\circ} \mathrm{C}$, Mesoscopic Physics Lab, Department of Geophysics, Colorado School of Mines, Golden (USA) 2009.

[2] F.P. Miller, A F. Vandome, J.M. Brewster, J. Phys. D: Appl. Phys. 42, 205501 (2009)

[3] H. Cetinkaya, A. Kaya, A. Unal, A. Vertiy, in: General Assembly and Scientific Symposium, 2011 XXXth URSI, Istanbul (Turkey)

[4] A.J. Devaney, IEEE Trans. Biomed. Eng. 30, 377 (1983)

[5] A. Vertiy, H. Cetinkaya, M. Tekbas, in: PIERS2010 Cambridge, Vol. 6, 2010, p. 485. 